\title{
Quantum oscillations in coupled orbits networks of (BEDT-TTF) salts with tris(oxalato)metallate anions
}

\author{
Vladimir N. Laukhin ${ }^{1,2}$, Alain Audouard ${ }^{3}$, Jean-Yves Fortin ${ }^{4}$, David Vignolles ${ }^{3}$, \\ Tatyana G. Prokhorova ${ }^{5}$, Eduard B. Yagubskii ${ }^{5}$ and Enric Canadell ${ }^{2}$ \\ ${ }^{1}$ Institució Catalana de Recerca i Estudis Avançats (ICREA), \\ Pg. Lluis Companys 23, 08010 Barcelona, Spain \\ ${ }^{2}$ Institut de Ciència de Materials de Barcelona, \\ Consejo Superior de Investigationes Cientificas, \\ Campus Universitat Autònoma de Barcelona, Bellaterra 08193, Spain \\ 3 Laboratoire National des Champs Magnétiques Intenses (UPR 3228 CNRS, \\ INSA, UGA, UPS) 143 avenue de Rangueil, F-31400 Toulouse, France \\ ${ }^{4}$ Institut Jean Lamour, Département de Physique de la Matière et des Matériaux, \\ CNRS-UMR 7198, Vandoeuvre-les-Nancy, F-54506, France \\ ${ }^{5}$ Institute of Problems of Chemical Physics, \\ Russian Academy of Sciences, 142432 Chernogolovka, MD, Russia
}

(Dated: June 28, 2016)

\begin{abstract}
Band structure calculations relevant to bis(ethylenedithio)tetrathiafulvalene-based charge transfer salts containing tris(oxalato)metallate anions, with generic formula (BEDT$\mathrm{TTF})_{4} \mathrm{~A}\left[\mathrm{M}\left(\mathrm{C}_{2} \mathrm{O}_{4}\right)_{3}\right] \cdot$ Solv, where $\mathrm{A}$ is a monovalent anion, $\mathrm{M}$ is a trivalent cation and Solv is a solvent, suggest that their Fermi surface is liable to achieve networks of compensated orbits coupled by magnetic breakdown. Even though this picture accounts for quantum oscillations spectra of a number of these compounds, puzzling results can be noticed in several cases, pointing to the possibility of Fermi surface reconstruction at low temperature.
\end{abstract}




\section{INTRODUCTION}

Even though the crystal structure of quasi-two-dimensional organic metals may appear complex compared to that of many inorganic compounds, their band structure is extremely simple. Indeed, according to calculations [1], the Fermi Surface (FS) of many salts, in particular those with $\beta$ " type packing considered hereafter, can be considered to originate from a hole orbit (labeled $\odot$ in the following) with an area equal to that of the first Brillouin zone (FBZ). Within an extended zone scheme, these orbits which can be approximated by ellipses, may overlap along either one or two directions, leading to either one- or two-dimensional, respectively, networks of orbits coupled by magnetic breakdown (MB) in magnetic fields [2] (see Fig. 1).

Quasi-two dimensional charge transfer salts with generic formula (BEDT$\mathrm{TTF})_{4}(\mathrm{~A})\left[\mathrm{M}\left(\mathrm{C}_{2} \mathrm{O}_{4}\right)_{3}\right] \cdot$ Solv (where BEDT-TTF stands for bis-ethylenedithiotetrathiafulvalene, $\mathrm{A}$ is a monovalent cation such as $\mathrm{H}_{3} \mathrm{O}^{+}, \mathrm{NH}_{4}^{+}$, etc., $\mathrm{M}$ is a trivalent cation such as $\mathrm{Fe}^{3+}, \mathrm{Cr}^{3+}$, etc. and Solv is a solvent such as dichlorobenzene $\left(\mathrm{C}_{6} \mathrm{H}_{4} \mathrm{Cl}_{2}\right)$, benzonitrile $\left(\mathrm{C}_{6} \mathrm{H}_{5} \mathrm{CN}\right)$, nitrobenzene $\left(\mathrm{C}_{6} \mathrm{H}_{5} \mathrm{NO}_{2}\right)$, etc.) set up a family of organic compounds which aroused great interest for many years [3] since the discovery of superconductivity in the iron-containing salt $\beta "$ " $(\mathrm{BEDT}-\mathrm{TTF})_{4}\left(\mathrm{H}_{3} \mathrm{O}\right) \mathrm{Fe}\left(\mathrm{C}_{2} \mathrm{O}_{4}\right)_{3} \cdot \mathrm{C}_{6} \mathrm{H}_{5} \mathrm{CN}$ [4].

Provided their ground-state is metallic, their FS topology is liable to illustrate the networks of orbits displayed in Fig. 1. For example, according to band structure calculations relevant to $\beta "-(\mathrm{BEDT}-\mathrm{TTF})_{4}\left(\mathrm{NH}_{4}\right)\left[\mathrm{Fe}\left(\mathrm{C}_{2} \mathrm{O}_{4}\right)_{3}\right] \cdot \mathrm{C}_{3} \mathrm{H}_{7} \mathrm{NO}$ [5] reported in Fig. 1(e) or $\beta "-(\mathrm{BEDT}-\mathrm{TTF})_{4} \mathrm{H}_{3} \mathrm{O}\left[\mathrm{Fe}\left(\mathrm{C}_{2} \mathrm{O}_{4}\right)_{3}\right] \cdot\left(2-\mathrm{C}_{5} \mathrm{H}_{4} \mathrm{BrN}\right)[6]$, the FS topology of these salts is similar to the scheme of Fig. 1(b) yielding one electron $(e)$ and one hole $(h)$ compensated orbits separated by small and large gaps, labeled $\Delta_{1}$ and $\Delta_{2}$, respectively. However, in the FBZ region around $\Delta_{2}$, the Fermi level is very close to the band extrema which can make the actual FS topology very sensitive to subtle structural details. Namely, if the gap is larger, the electron-type orbits transform into quasi-one dimensional sheets as displayed in Fig. 1(a). Oppositely, in the case where the gap is closed, $\odot$ orbits intersect along two directions, yielding the scheme of Fig. 1(c), with two hole $\left(h_{1}\right.$ and $\left.h_{2}\right)$ and one electron $(e)$ compensated orbits. This is illustrated by e.g. band structure calculations relevant to the compound $(\mathrm{BEDO})_{4} \mathrm{Ni}(\mathrm{CN})_{4} \cdot 4 \mathrm{CH}_{3} \mathrm{CN}[7]$, where BEDO stands for the bis-ethylenedioxi-tetrathiafulvalene molecule. More recently, compounds with two different 
alternating BEDT-TTF layers have been further synthesized [8, 9] such as $\alpha$-'pseudo- $\kappa$ '$(\mathrm{BEDT}-\mathrm{TTF})_{4} \mathrm{H}_{3} \mathrm{O}\left[\mathrm{Fe}\left(\mathrm{C}_{2} \mathrm{O}_{4}\right)_{3}\right] \cdot\left(\mathrm{C}_{6} \mathrm{H}_{4} \mathrm{Br}_{2}\right)$. Whereas, layers with 'pseudo- $\kappa$ ' structure are insulating, $\alpha$ layers, the FS of which is depicted in Fig. 1(d), are metallic. In the latter case, due to folding of the FBZ, the FS is composed of two electron and one hole compensated orbits. At variance with the above considered cases, these three orbits are connected by a MB junction whereas the set is isolated within the FBZ $[8,10]$.

According to the above statements, all these compounds may offer a playground for the study of quantum oscillations in networks of orbits. Nevertheless, puzzling experimental data can be observed, in line with the various reported ground-states, including normal metal, charge density wave, superconductivity, and temperature-dependent behaviours.

In this paper we review FS parameters (frequencies, effective masses) and Fourier amplitude calculations in coupled orbits networks (Section II). In Section III, recent data concerning quantum oscillations in the family of BEDT-TTF molecular metals containing tris(oxalato)metallate anions are examined at the light of these calculations.

\section{CALCULATION OF FERMI SURFACE PARAMETERS}

In this section, we consider frequencies, effective masses and Fourier amplitudes relevant to the various FS topologies presented in Fig. 1. The case of $\odot$ orbits overlapping in one direction, illustrated in Fig. 1(a), is nevertheless put aside since it has not been reported for the considered family yet. Let us simply recall that it corresponds to the widely studied linear chain of coupled (hole) orbits (see e.g. $[2,11]$ and references therein) for which the two basic frequencies observed in the oscillatory spectra correspond to the closed $h$ and MB-induced $\odot$ orbits, known as the $\alpha$ and $\beta$ orbits, respectively, in the literature. Fourier spectra, which are composed of linear combinations of these two frequencies $\left(F_{\alpha} \sim 500-1000\right.$ $\mathrm{T}, F_{\beta} \approx 4000 \mathrm{~T}$ ), are strongly influenced by oscillations of the chemical potential in magnetic field.

For $\odot$ orbits either coming close together in the direction transverse with respect to the chain or intersecting in two directions (see schemes of Figs. 1(b) and (c), respectively) compensated orbits are observed. In contrast with the above mentioned case, oscillations of the chemical potential are significantly damped $[12,13]$ and the Lifshitz-Kosevich formalism [14-16] holds. 

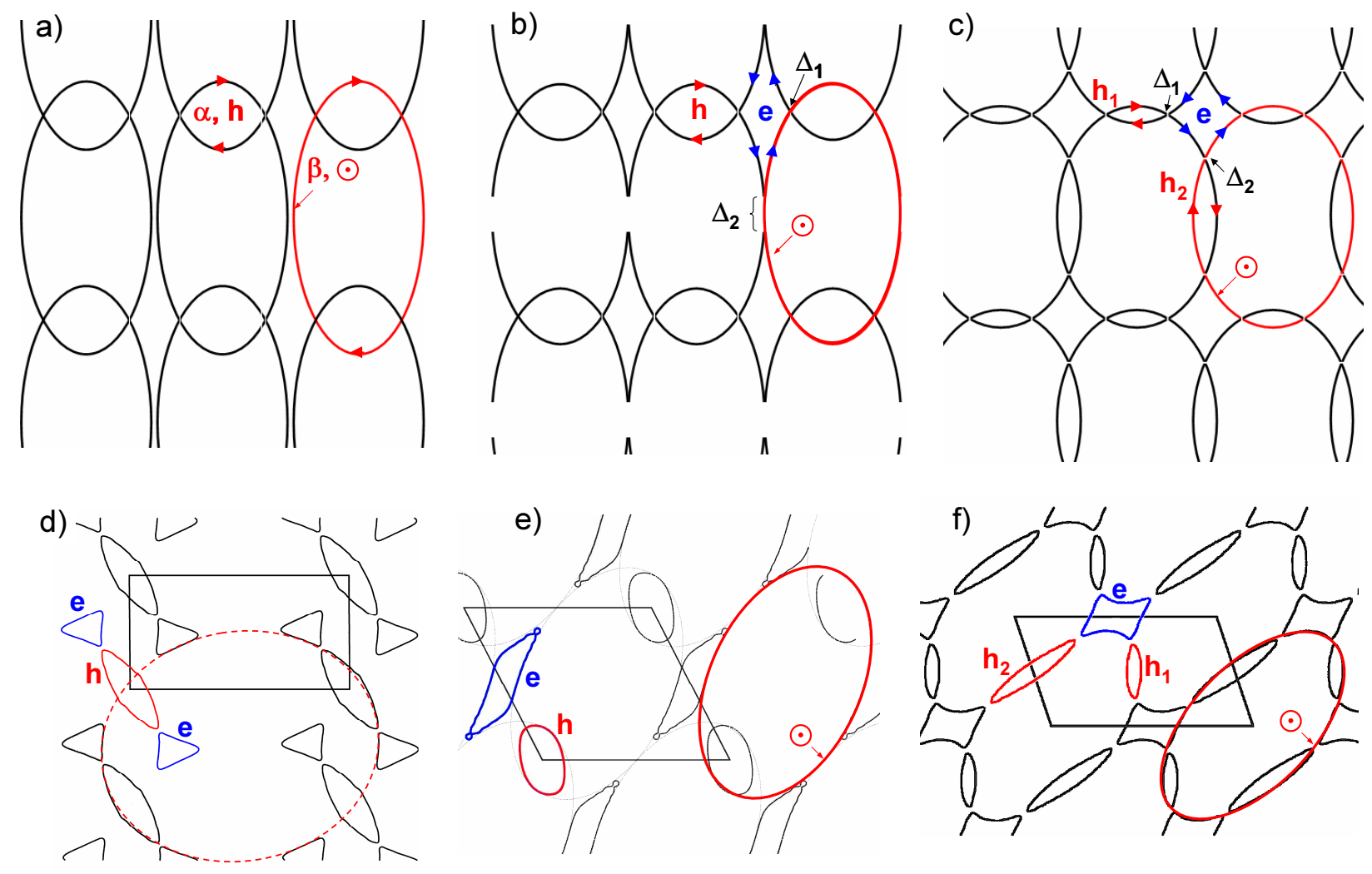

FIG. 1: Sketch of Fermi surfaces formed by overlapping of elliptic $\odot$ orbits along (a) one and (c) two directions. An intermediate case in which the $\odot$ orbits come close to each other in one direction is depicted in (b). Fermi surface calculations of (d) $\alpha$-'pseudo- $\kappa$ '-(BEDTTTF $)_{4} \mathrm{H}_{3} \mathrm{O}\left[\mathrm{Fe}\left(\mathrm{C}_{2} \mathrm{O}_{4}\right)_{3}\right] \cdot\left(\mathrm{C}_{6} \mathrm{H}_{4} \mathrm{Br}_{2}\right)$ [10], (e) $\beta "$ - $(\mathrm{BEDT}-\mathrm{TTF})_{4}\left(\mathrm{NH}_{4}\right)\left[\mathrm{Fe}\left(\mathrm{C}_{2} \mathrm{O}_{4}\right)_{3}\right] \cdot \mathrm{C}_{3} \mathrm{H}_{7} \mathrm{NO}$ [5] and (f) $(\mathrm{BEDO})_{4} \mathrm{Ni}(\mathrm{CN})_{4} \cdot 4 \mathrm{CH}_{3} \mathrm{CN}[7]$. (e) and (f) are illustrations of (b) and (c), respectively. Red and blue labels correspond to hole and electron orbits, respectively. Arrows indicate direction of quasiparticles. Ellipses in solid red lines have an area equal to that of the first Brillouin zone and correspond to the $\odot$ orbits. Ellipse in dashed line in $(\mathrm{d})$ has an area twice the First Brillouin zone due to folding.

Within this formalism, for magnetic field applied parallel to the normal to the conducting plane, oscillatory part of the magnetization relevant to the harmonic $\lambda$ of an orbit $\eta$ can be written

$$
m_{\text {osc }}=-\sum_{\eta} \sum_{\lambda} A(\eta, \lambda) \sin \left(2 \pi \lambda \frac{F_{\eta}}{B}\right) .
$$

for two-dimensional FS. The de Haas-van Alphen amplitude $A(\eta, \lambda)$ is given by 

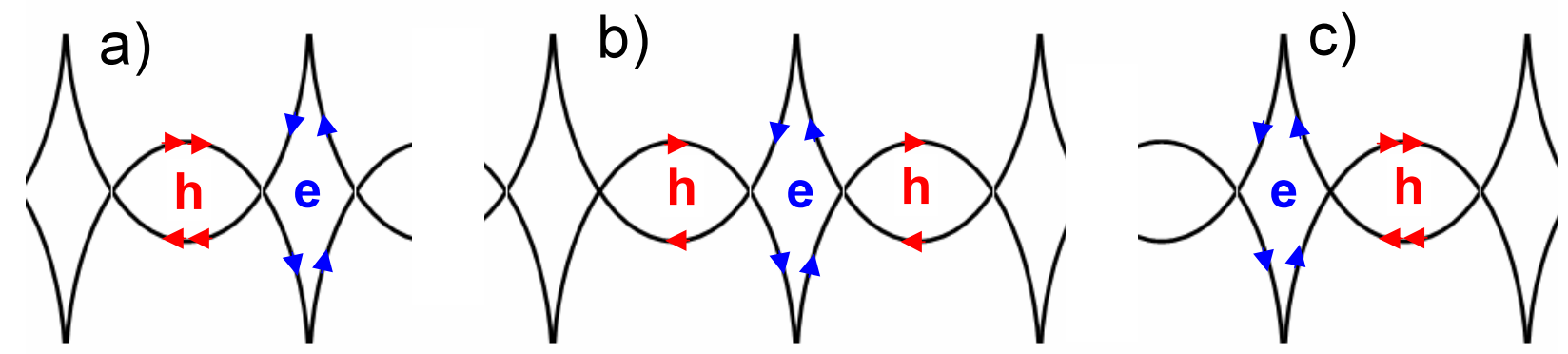

FIG. 2: Examples of magnetic breakdown (MB) orbits, relevant to the linear chain of compensated orbits of Fig. 1(b), involving 1 electron and 2 hole orbits. These three orbits yield the same frequency corresponding to one single orbit and have the same effective mass, $m=m_{e}+2 m_{h}$. However, MB damping factors are different: $p^{2}\left(1-p^{2}\right)^{2}$ for (a) and (c), and $p^{4}\left(1-p^{2}\right)$ for (b), where $p=\exp \left(-B_{0} / 2 B\right)$ is the tunneling probability and $B_{0}$ is the MB field.

$$
A(\eta, \lambda)=(-1)^{\lambda s_{\eta}} \frac{F_{\eta}}{\pi \lambda m_{\eta}} R(\eta, \lambda) R_{\eta, \lambda}^{M B}
$$

where $2 s_{\eta}$ is the number of turning points of the orbit and $R(\eta, \lambda)=R_{\eta, \lambda}^{T} R_{\eta, \lambda}^{D} R_{\eta, \lambda}^{s}$. The temperature, Dingle and spin damping factors are given by [16] $R_{\eta, \lambda}^{T}=X_{\eta, \lambda} \sinh ^{-1}\left(X_{\eta, \lambda}\right)$, $R_{\eta, \lambda}^{D}=\exp \left(-u_{0} \lambda m_{\eta} T_{D \eta} / B\right)$ and $R_{\eta, \lambda}^{s}=\cos \left(\pi g_{\eta} \lambda m_{\eta} / 2\right)$, respectively, where the fieldand temperature-dependent variable $\left(X_{\eta, \lambda}\right)$ and the constant $\left(u_{0}\right)$ are expressed as $X_{\eta, \lambda}$ $=u_{0} \lambda m_{\eta} T / B$ and $u_{0}=2 \pi^{2} k_{B} m_{0}(e \hbar)^{-1}=14.694 \mathrm{~T} / \mathrm{K} . T_{D \eta}, m_{\eta}$ and $g_{\eta}$ are the Dingle temperature, effective mass in $m_{0}$ units and effective Landé factor, respectively. In the following, the $\lambda^{\text {th }}$ harmonic of $\eta$ is regarded as the orbit $\lambda \eta$ since $R(\eta, \lambda)=R(\lambda \eta, 1)$. The MB damping factor is $R_{\eta, \lambda}^{M B}=C_{\eta, \lambda}(i p)^{n^{t}}(q)^{n^{r}}$ where $C_{\eta, \lambda}$ is the symmetry factor. The tunneling $(p)$ and reflection $(q)$ probabilities are given by $p=e^{-B_{0} / 2 B}$ and $p^{2}+q^{2}=1$ where $B_{0}$ is the MB field [17]. For magnetic fields tilted by an angle $\theta$ from the normal to the conducting plane, the above formulas still hold provided $B$ is changed to $B \cos (\theta)$.

\section{A. One-dimensional chain of compensated orbits}

In the case of the scheme of Fig. 1(b), which is illustrated by the band structure calculations of Fig. 1(e), the FS is composed of a pair of compensated electron $(e)$ and hole (h) orbits. Provided the MB gap $\Delta_{2}$ in the direction parallel to the chains is large enough, 
a linear chain of compensated orbits is obtained. The primary point is that, owing to the 'opposite sign' of electron and hole orbits, any $\eta$ orbit composed of $n_{e}$ electron and $n_{h}$ hole orbits corresponds to the Fourier component with frequency $F_{\delta n}=\delta n F_{1}$, where the fundamental frequency $F_{1}$ is the frequency linked to both $e$ and $h$ orbits $\left(F_{1}=F_{e}=F_{h}\right)$ and $\delta n$ $=\left|n_{e}-n_{h}\right|$. In the framework of the Falicov-Stachowiak model $[16,18]$, the effective mass is given by $m_{\eta}=n_{e} m_{e}+n_{h} m_{h}$, where $m_{e(h)}$ is the absolute value of the effective mass of individual electron (hole) orbit. As a result, an infinite number of $\eta$ orbits corresponds to the frequency $F_{\delta n}$. Moreover, $\eta$ orbits with the same number of $e$ and $h$ orbits may have different MB damping factors. This point is exemplified in Fig. 2 in which the case of three $\eta$ orbits composed of one electron and two hole orbits $(\eta=e+2 h)$ is considered. Whereas they correspond to the same frequency $F_{1}$ and have the same effective mass $m_{\eta}=m_{e}+2 m_{h}$, their MB damping factors are different: $p^{2}\left(1-p^{2}\right)^{2}$ for Figs. $2(\mathrm{a})$ and (c), and $p^{4}\left(1-p^{2}\right)$ for Fig. 2(b). Extensive calculation of the Fourier amplitude, based on combinatorial analysis, has been reported in [13]. Limiting ourselves to $\eta$ orbits with frequency $F_{1}$ made of at most three individual orbits, yields:

$$
A_{1}=A_{e}+A_{h}+A_{2 e+h}+A_{e+2 h}+\cdots
$$

with

$$
\begin{array}{r}
A_{e}=-\frac{F_{1}}{m_{e}} R_{e}\left(1-p^{2}\right) \\
A_{h}=-\frac{F_{1}}{m_{h}} R_{h}\left(1-p^{2}\right) \\
A_{2 e+h}=\frac{F_{1}}{2 m_{e}+m_{h}} R_{2 e+h}\left[p^{2}\left(1-p^{2}\right)^{2}\left(2-3 p^{2}\right)\right] \\
A_{e+2 h}=\frac{F_{1}}{2 m_{h}+m_{e}} R_{e+2 h}\left[p^{2}\left(1-p^{2}\right)^{2}\left(2-3 p^{2}\right)\right]
\end{array}
$$

As a consequence, the effective mass deduced from temperature dependence of the amplitude $A_{1}$ depends on many parameters, namely on the relative value of $m_{e}$ and $m_{h}$, Dingle temperature, MB field and effective Landé factors. For strongly different $m_{e}$ and $m_{h}$ values, $A_{1}$ is governed by the smaller effective mass, provided the contributions of $e+2 h$ and $2 e+h$ are small enough. In contrast, as $m_{e}$ and $m_{h}$ are close, a mean value is deduced from experimental data. Besides, for small $B_{0} / B$ values, hence large tunneling probability, orbits $2 e+h$ and $e+2 h$ can contribute substantially, leading to non Lifshitz-Kosevich behaviour (i.e. 
apparent temperature-dependent effective mass) [13]. In addition, $A_{1}$ is strongly dependent on the relative values of the spin damping factors which are governed by the product $g_{\eta} m_{\eta}$. Owing to this statement, experiments with various directions of the magnetic field with respect to the conducting plane can yield more insight on the involved parameters.

\section{B. Two-dimensional network of compensated orbits}

Analogous considerations hold for the two-dimensional network of Fig. 1(c). It must be noticed that such network which is illustrated by the band structure calculations of Fig. 1(f), has not been predicted for the considered family (i.e. with BEDT-TTF as the donor), yet. Nevertheless, as discussed in the next Section, several experimental oscillatory spectra are in line with this picture. In this case, the FS is composed of one electron $(e)$ and two hole $\left(h_{1}\right.$ and $\left.h_{2}\right)$ orbits. Therefore, instead of one Fourier peak and harmonics, spectra are composed of linear combinations of components with frequencies $F_{e}, F_{h 1}$ and $F_{h 2}$. Since orbits compensation yields the additional relationship $F_{e}=F_{h 1}+F_{h 2}$, frequency $F\left(n_{e}, n_{h 1}, n_{h 2}\right)$ resulting from an $\eta$ orbit made of $n_{e}, n_{h 1}$ and $n_{h 2}$ orbits is given by $F\left(n_{e}, n_{h 1}, n_{h 2}\right)=$ $\left|\left(n_{e}-n_{h 2}\right) F_{e}+\left(n_{h 2}-n_{h 1}\right) F_{h 1}\right|$. The exception of $\eta=n_{h 1} h_{1}+n_{h 2} h_{2}$ must nevertheless be noticed since any MB orbit solely made with hole orbits cannot exist according to Fig. 2(c). Therefore, as it is the case for the one-dimensional chain, a given frequency results from the contribution of an infinite number of $\eta$ orbits. As an example, the frequency $F_{h 1(2)}$ results from the contribution of the orbits $e+h_{2(1)}, h_{1(2)}, 2 e+h_{1(2)}+2 h_{2(1)}, 3 e+2 h_{1(2)}+3 h_{2(1)}$, etc., yielding, limiting ourselves to the two first $\eta$ orbits with the lowest effective masses:

$$
A\left(F_{h 1(2)}\right)=A_{h 1(2)}+A_{e+h 2(1)}+\cdots
$$

with, taking into account the symmetry factors $\left(1\right.$ and 2 for $h_{1(2)}$ and $e+h_{2(1)}$, respectively),

$$
\begin{aligned}
& A_{h 1(2)}=-\frac{F_{1}}{m_{h 1(2)}} R_{h 1(2)}\left(1-p_{1(2)}^{2}\right) \\
& A_{e+h 2(1)}=-\frac{F_{1}}{2 m_{h}+m_{e}} R_{e+2 h}\left[2 p_{2(1)}^{2}\left(1-p_{2(1)}^{2}\right)\left(1-p_{1(2)}^{2}\right)\right]
\end{aligned}
$$

where $p_{1(2)}=\exp \left(-B_{0,1(2)} / B\right)$ and $B_{0,1(2)}$ is the MB field linked to the gap $\Delta_{1(2)}$. 
Similarly, in addition to the $e$ orbit, more complex MB orbits such as $e+2 h_{1}+2 h_{2}$, $2 e+2 h_{1}+2 h_{2}, 2 e+3 h_{1}+3 h_{2}$, etc. albeit with large effective mass and small MB damping factors, can contribute to $F_{e}$. In that respect, more extensive calculations, such as reported in Ref.[13] for the one-dimensional chain, are needed to get a more deeper insight on the oscillatory behaviour of two-dimensional networks of compensated orbits. Nevertheless, it can be considered that for large enough Dingle temperature and MB gaps only orbits with the smallest effective masses can significantly contribute to the data.

\section{Isolated set of compensated orbits}

Finally, the case of isolated set made of two electron and one hole compensated orbits reported in Fig. 1(d) has been considered in Ref. [10]. Owing to orbits compensation, leading to $F_{h}=2 F_{e}$, oscillation spectra are composed of a series of Fourier components with frequencies $\left|n_{e}-2 n_{h}\right| F_{e}$ and effective masses $n_{e} m_{e}+n_{h} m_{h}$ where $n_{e(h)}$ is the number of involved electron (hole) orbits. As a result, each Fourier component results from many MB orbits. E.g., the two main frequencies, $F_{e}$ and $F_{h}=2 F_{e}$, arise from $e, e+h, 3 e+h, 3 e+2 h$, etc. and $2 e, h, 4 e+h, 2 e+2 h$, etc., respectively. Their amplitudes are given by:

$$
A_{e}=-\frac{2\left(1-p^{2}\right)^{1 / 2}}{m_{e}} R_{e}-\frac{2 p^{2}\left(1-p^{2}\right)^{1 / 2}}{m_{e}+m_{h}} R_{e+h}+\frac{2\left[p^{4}\left(1-p^{2}\right)^{1 / 2}-p^{2}\left(1-p^{2}\right)^{3 / 2}\right]}{3 m_{e}+m_{h}} R_{3 e+h}+\cdots
$$

and

$$
\begin{gathered}
A_{2 e}=\frac{\left(1-p^{2}\right)}{2 m_{e}} R_{2 e}-\frac{\left(1-p^{2}\right)}{m_{h}} R_{h}+\frac{2 p^{2}\left(1-p^{2}\right)^{2}-3 p^{4}\left(1-p^{2}\right)^{2}}{4 m_{e}+m_{h}} R_{4 e+h} \\
+\frac{3 p^{4}\left(1-p^{2}\right)-2 p^{2}\left(1-p^{2}\right)^{2}}{2\left(m_{e}+m_{h}\right)} R_{2 e+2 h}+\cdots .
\end{gathered}
$$

\section{COMPARISON WITH EXPERIMENTAL DATA}

In this section, experimental data reported in the literature are examined at the light of the above calculations. To our best knowledge, and as far as high magnetic field data relevant to salts with tris(oxalato)metallate anions are concerned, the one-dimensional chain of compensated orbits and the isolated set of compensated orbits considered in Section II A and II C, respectively, are only illustrated by $\beta$ " - $(\mathrm{BEDT}-\mathrm{TTF})_{4} \mathrm{H}_{3} \mathrm{O}\left[\mathrm{Cr}\left(\mathrm{C}_{2} \mathrm{O}_{4}\right)_{3}\right] \cdot \mathrm{C}_{6} \mathrm{H}_{5} \mathrm{NO}_{2}$ 
TABLE I: Frequencies and effective masses reported in the literature for (BEDT$\mathrm{TTF})_{4} \mathrm{~A}\left[\mathrm{M}\left(\mathrm{C}_{2} \mathrm{O}_{4}\right)_{3}\right] \cdot$ Solv, labelled as AM.Solv, where DMF and DCB stand for $\mathrm{C}_{3} \mathrm{H}_{7} \mathrm{NO}$ and $\mathrm{C}_{6} \mathrm{H}_{4} \mathrm{Cl}_{2}$, respectively. Effective mass of the $\odot$ orbit is calculated as $m_{\odot}=m_{e}+m_{h 1}+m_{h 2}$

\begin{tabular}{lcccccccc}
\hline compound & $F_{a}$ & $F_{b-a}$ & $F_{b}$ & $F_{a}+F_{b-a}$ & $m_{a}$ & $m_{b-a}$ & $m_{b}$ & $m_{\odot}$ \\
\hline$\left(\mathrm{NH}_{4}\right) \mathrm{Fe} \cdot \mathrm{DMF}[30]$ & $48(2)$ & $192(2)$ & $241(5)$ & $240(4)$ & $0.58(6)$ & $0.7(1)$ & $1.07(10)$ & $2.35(26)$ \\
$\left(\mathrm{NH}_{4}\right) \mathrm{Cr} \cdot \mathrm{DMF}(0.8 \mathrm{GPa})[29]$ & 67 & 245 & 311 & 312 & $0.4(2)$ & $0.80(25)$ & $1.10(25)$ & $2.30(65)$ \\
$\left(\mathrm{NH}_{4}\right) \mathrm{Cr} \cdot \mathrm{DMF}(1 \mathrm{GPa})[29]$ & 68 & 255 & 320 & 323 & $0.60(15)$ & $0.8(2)$ & $0.8(2)$ & $2.20(55)$ \\
$\left(\mathrm{H}_{3} \mathrm{O}\right) \mathrm{Fe} \cdot \mathrm{DCB}[26,28]$ & $74(5)$ & $272(5)$ & $348(3)$ & $346(10)$ & $0.85(10)$ & $1.0(1)$ & $1.5(1)$ & $3.35(30)$ \\
\hline
\end{tabular}

[19] and $\alpha$-'pseudo- $\kappa$ '-(BEDT-TTF $)_{4} \mathrm{H}_{3} \mathrm{O}\left[\mathrm{Fe}\left(\mathrm{C}_{2} \mathrm{O}_{4}\right)_{3}\right] \cdot\left(\mathrm{C}_{6} \mathrm{H}_{4} \mathrm{Cl}_{2}\right)$ [8], respectively. In the former case, only one frequency $F=230(10) \mathrm{T}$, corresponding to a rather small area of about 6 $\%$ of the FBZ area, is observed. This result supports the scheme of Fig. 1(b) and band structure calculations relevant to $\beta "-(\mathrm{BEDT}-\mathrm{TTF})_{4} \mathrm{NH}_{4}\left[\mathrm{Fe}\left(\mathrm{C}_{2} \mathrm{O}_{4}\right)_{3}\right] \cdot \mathrm{C}_{3} \mathrm{H}_{7} \mathrm{NO}[5]$ and $\beta "-(\mathrm{BEDT}-$ $\left.\mathrm{TTF})_{4} \mathrm{H}_{3} \mathrm{OFe}\left(\mathrm{C}_{2} \mathrm{O}_{4}\right)_{3}\right] \cdot\left(\mathrm{C}_{6} \mathrm{H}_{5} \mathrm{CN}\right)_{0.35}\left(\mathrm{C}_{6} \mathrm{H}_{5} \mathrm{Cl}\right)_{0.65}[9]$, which predict FS areas of the same order of magnitude ( $8.8 \%$ and $7.4 \%$ of the FBZ area, respectively). Considering the small orbit area, rather high effective mass is obtained $\left(m^{*}=2.2\right)$ attributed to the presence of $\mathrm{Cr}^{3+}$ magnetic ions. Nevertheless, since the MB field is not known, hampering comprehensive data analysis on the basis of Eqs. 3-7, it is not possible to infer the respective effective mass of electron and hole orbits. In contrast, field, temperature and angle dependance of the oscillations spectrum are reported for $\alpha$-'pseudo- $\kappa$ '-(BEDT-TTF $)_{4} \mathrm{H}_{3} \mathrm{O}\left[\mathrm{Fe}\left(\mathrm{C}_{2} \mathrm{O}_{4}\right)_{3}\right] \cdot\left(\mathrm{C}_{6} \mathrm{H}_{4} \mathrm{Cl}_{2}\right)$ [10]. Despite the observed rather large Dingle temperatures, the orbit $e+h$ has a definite influence on the amplitude $\left(A_{e}\right)$ of the oscillations with frequency $F_{e}$ due to a small MB gap $\left(B_{0} \sim 2 \mathrm{~T}\right)$, whereas, albeit to a lesser extent, $2 e$ contribute to the amplitude $A_{h}$. In short, the data support Eqs. 11 and 12, allowing the determination of the effective masses $m_{e}=0.93(4)$ and $m_{h}=0.88(4)$ which are rather close to each other even though the $h$ orbit area is by a factor of two larger than the $e$ orbits area. Finally, beyond the case of salts with tris(oxalato)metallate anions, it can be remarked that such FS topology also holds for (BEDO-TTF) ${ }_{2} \mathrm{ReO}_{4} \cdot \mathrm{H}_{2} \mathrm{O}[20]$ whose quantum oscillations have been studied in e.g. Refs. [21, 22] and for which similar data analysis would be important to unravel the so far not adequately understood quantum oscillations behaviour in this salt. 

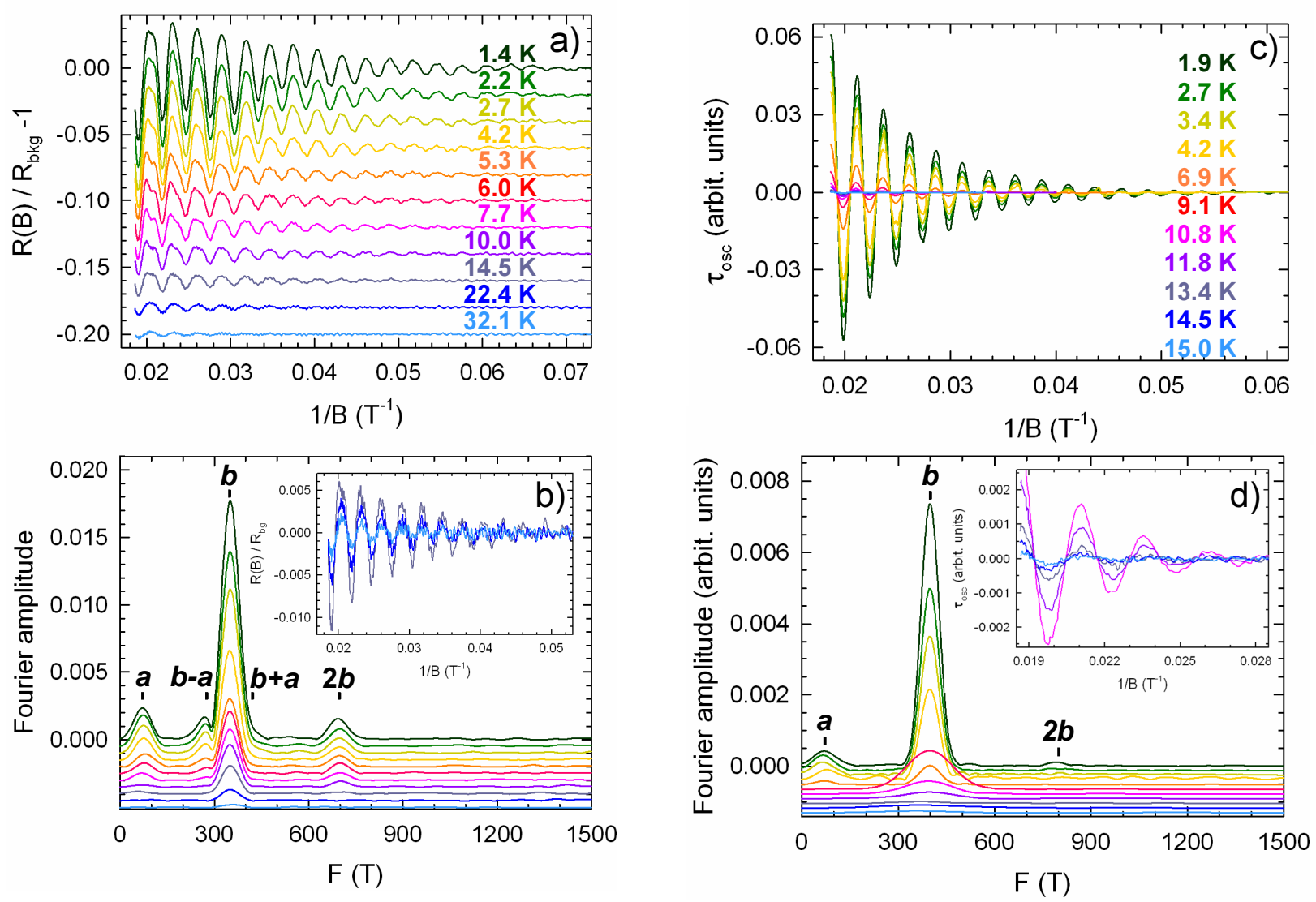

FIG. 3: Oscillatory data relevant to $\beta$ "-(BEDT-TTF $)_{4} \mathrm{H}_{3} \mathrm{O}\left[\mathrm{Fe}\left(\mathrm{C}_{2} \mathrm{O}_{4}\right)_{3}\right] \cdot \mathrm{C}_{6} \mathrm{H}_{4} \mathrm{Cl}_{2}$. (a) Shubnikovde Haas oscillations and (c) magnetic torque data (yielding de Haas-van Alphen oscillations). Corresponding Fourier analysis are given in (b) and (d), respectively (from [28]).

Unlike the two cases above, many experimental data are, at first sight, in line with the scheme of Fig. 1(c). As examples, combinations of frequencies predicted in Section II B for two-dimensional networks are observed for the data reported in Table I: for all the considered compounds, three frequencies, labeled $F_{a}, F_{b-a}$ and $F_{b}$ are observed. Within the reported uncertainties, the relationship $F_{a}+F_{b-a}=F_{b}$ holds, in line with the considered type of two-dimensional network, provided it is assumed that the frequencies $F_{a}$ and $F_{b-a}$ are linked to the hole orbits $\left(h_{1}\right.$ and $h_{2}$ ) while $F_{b}$ corresponds to the electron orbit. Within this framework, the electron orbit area amounts to 6 to $9 \%$ of the FBZ area.

Effective mass of the $\odot$ orbit (see Fig. 1) can be estimated as $m_{\odot}=m_{e}+m_{h 1}+m_{h 2}$. Assuming as a tentative approximation that $m_{b}, m_{a}$ and $m_{b-a}$ correspond to the effective masses of the orbits $m_{e}, m_{h 1(2)}$ and $m_{h 2(1)}$ (i.e. the complex MB orbits mentioned in Sec- 
tion II B, liable to enter the relevant amplitudes, and MB gaps have negligible contributions) yield $m_{\odot} \sim 2-4$ which is within the reported values for compounds with the FS topology of Fig. 1(a), for which the effective mass of the $\beta$ orbit (equivalent to the $\odot$ orbit) ranges from $m_{\beta}=1.9(1)$ for $\theta$-(BEDT-TSF) ${ }_{4} \mathrm{CoBr}_{4} \cdot\left(\mathrm{C}_{6} \mathrm{H}_{4} \mathrm{Cl}_{2}\right)$ [23] (where BEDT-TSF stands for the bis-ethylenedithio-tetraselenofulvalene molecule) to $m_{\beta}=6 \sim 7$ for the starring compound $\kappa-(\mathrm{BEDT}-\mathrm{TTF})_{2} \mathrm{Cu}(\mathrm{SCN})_{2}[24,25]$.

Nevertheless, we have to be cautious with the physical meaning of the parameters determined from experimental data. Indeed, let us consider $\beta$ "-(BEDT$\mathrm{TTF})_{4} \mathrm{H}_{3} \mathrm{O}\left[\mathrm{Fe}\left(\mathrm{C}_{2} \mathrm{O}_{4}\right)_{3}\right] \cdot \mathrm{C}_{6} \mathrm{H}_{4} \mathrm{Cl}_{2}$ which is the most extensively studied compound of this family, with respect to quantum oscillations [26-28]. Whereas Shubnikov-de Haas (SdH) spectra evidence the three frequencies $F_{a}, F_{b-a}$ and $F_{b}$, in line with the considered scheme (see Table I), de Haas-van Alphen spectra, obtained with a tilt angle $\theta=29^{\circ}$, only evidence $F_{a}$ and $F_{b}$ as reported in Fig. 3. Unless spin-zero phenomenon could be invoked to explain the absence of $F_{b-a}$, this feature is unexplained. In that respect, any angle dependence study of the $b-a$ component is unfortunately not reported, yet. Besides, even though the temperature dependence of the dHvA $b$ oscillations amplitude follows the Lifshitz-Kosevich behaviour, a strong slowing down of the temperature-dependent damping of the $\mathrm{SdH}$ amplitude is observed at high temperature (see Fig. 4). As a result, $b$ oscillations are observed up to $32 \mathrm{~K}$. This feature is consistent with a zero-effective mass quantum interferometer. However, not any quantum interferometer can be obtained within the considered two-dimensional network.

Even more obvious discrepancies between the model FS of Fig. 1(c) and quantum oscillations data are observed in the literature. For example, even though $\mathrm{SdH}$ data of $\beta$ "$(\mathrm{BEDT}-\mathrm{TTF})_{4} \mathrm{NH}_{4}\left[\mathrm{Cr}\left(\mathrm{C}_{2} \mathrm{O}_{4}\right)_{3}\right] \cdot \mathrm{C}_{3} \mathrm{H}_{7} \mathrm{NO}$, collected under applied pressures of $0.8 \mathrm{GPa}$ and $1 \mathrm{GPa}$, can be interpreted on the basis of three compensated orbits as above [29], up to six Fourier components with no clear frequency combinations are observed at ambient pressure [29]. In contrast, substituting $\mathrm{Cr}^{3+}$ for $\mathrm{Fe}^{3+}$ allow to recover the three frequencies $F_{a}, F_{b-a}$ and $F_{b}$ at ambient pressure and low temperature (see Table I and Ref. [30]). However, an unexplained splitting of the $b$ component is observed above $\sim 3 \mathrm{~K}$ in this compound. In addition, even though the low temperature spectrum is preserved under applied pressure of up to $0.98 \mathrm{GPa}$, an unexplained huge pressure-induced increase of the frequencies is observed $\left(d(\ln (F)) / d P=1 G P a^{-1}\right)[31]$. To end with this series of puzzling examples, $\beta$ "- 


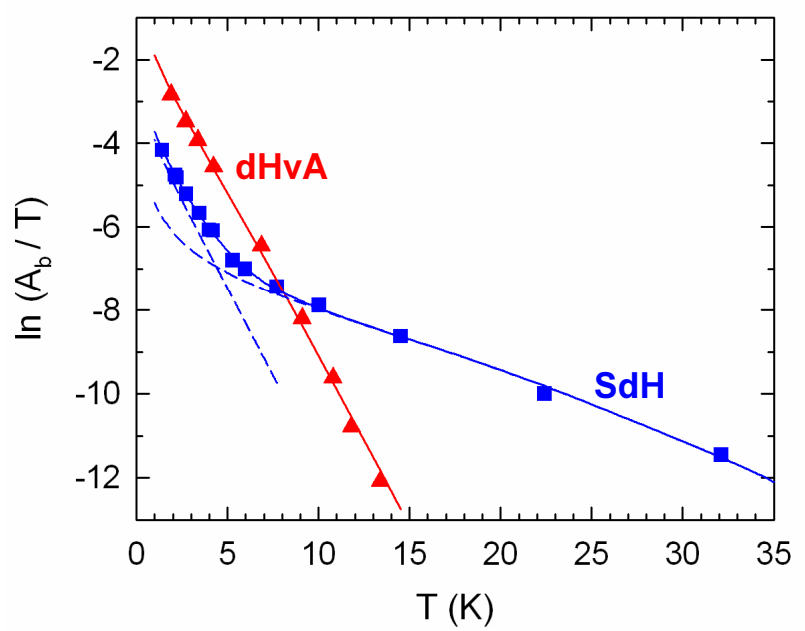

FIG. 4: Temperature dependence of the $b$ oscillations amplitude deduced from data of Fig. 3. Solid red and blue lines are the best fits of the LK model assuming that either only one closed orbit contributes to the amplitude ( $\mathrm{dHvA}$ data) or a closed orbit and a quantum interferometer, with zero effective mass and a temperature-dependent scattering rate, coexist ( $\mathrm{SdH}$ data), respectively. These two latter contributions are displayed as dashed curves. From Ref. [28].

$(\mathrm{BEDT}-\mathrm{TTF})_{4} \mathrm{H}_{3} \mathrm{O}\left[\mathrm{M}\left(\mathrm{C}_{2} \mathrm{O}_{4}\right)_{3}\right] \cdot \mathrm{C}_{5} \mathrm{H}_{5} \mathrm{~N}_{2}$ salts, with $\mathrm{M}=\mathrm{Ga}, \mathrm{Cr}$, Fe, reveal two main Fourier components with frequencies in the range 38 to $50 \mathrm{~T}$ and $340 \sim 350 \mathrm{~T}(\sim 8.5 \%$ of the FBZ area), respectively [32]. In addition to these frequencies which are close to $F_{a}$ and $F_{b}$, respectively, a plethora of Fourier components are observed. Few of them could be considered as frequency combinations. In the case of the scheme depicted in Fig. 1(a) involving only hole orbits, oscillations of the chemical potential can induce frequency combinations $[2,10,25]$. However, this phenomenon can be barely invoked for compensated multiband metals [13]. As a result, it is likely that the observed frequencies actually corresponds to classical orbits which rules out the textbook scheme of Fig. 1(c). Phase transition such as density wave condensation can be invoked to interpret these discrepancies [33] since they can drastically change the FS topology at low temperature.

\section{SUMMARY AND CONCLUSION}

According to band structure calculations, many charge transfer salts with generic formula $\beta "$ - $(\mathrm{BEDT}-\mathrm{TTF})_{4} \mathrm{~A}\left[\mathrm{M}\left(\mathrm{C}_{2} \mathrm{O}_{4}\right)_{3}\right] \cdot$ Solv are two-dimensional compensated metals. Accordingly, 
they are liable to illustrate textbook Fermi surfaces predicted by band structure calculations hence providing a playground for the study of quantum oscillations. Field- and temperaturedependent de Haas-van Alphen oscillations amplitude are evaluated for the one-dimensional chain and the two-dimensional network of compensated orbits. However, although the observed frequencies are in agreement with predictions of band structure calculations in several cases, either comprehensive experimental data are missing or the behaviour of the amplitudes is not in agreement with calculations, hence with predicted band structures, pointing to a Fermi surface reconstruction at low temperature in these compounds. As a result, comprehensive data illustrating one- and two-dimensional networks of orbits are still needed. A noticeable exception is provided by $\alpha$-'pseudo- $\kappa$ '- $(\mathrm{BEDT}-\mathrm{TTF})_{4} \mathrm{H}_{3} \mathrm{O}\left[\mathrm{Fe}\left(\mathrm{C}_{2} \mathrm{O}_{4}\right)_{3}\right] \cdot\left(\mathrm{C}_{6} \mathrm{H}_{4} \mathrm{Cl}_{2}\right)$ the oscillations data of which are quantitatively accounted for by a Fermi surface made of an isolated set of orbits.

\section{Acknowledgments}

The support of the European Magnetic Field Laboratory (EMFL) is acknowledged. V.N.L. and E.C. acknowledge support of the Spanish MINECO through the Severo Ochoa Centers of Excellence Program under Grant SEV-2015-0496. This work has been supported by MINECO through Grant FIS2015-64886-C5-4-P and Generalitat de Catalunya (2014SGR301). It was also supported by the Russian Foundation for Basic Research, project No 14-03-0019 and the Program No.2 of Presidium of Russian Academy Sciences.

[1] R. Rousseau, M. Gener and E. Canadell, Adv. Funct. Mater. 14201 (2004).

[2] A. Audouard and J.-Y. Fortin, C. R. Physique 1415 (2013).

[3] E. Coronado and P. Day, Chem. Rev. 1045419 (2004).

[4] M. Kurmoo, A. W. Graham, P. Day, S. J. Coles, M. B. Hursthouse, J. L. Caulfield, J. Singleton, F. L. Pratt, W. Hayes, L. Ducasse and P. Guionneau, J. Am. Chem. Soc. 11712209 (1995).

[5] T. G. Prokhorova, S. S. Khasanov, L. V. Zorina, L. I. Buravov, V. A. Tkacheva, A. A. Baskakov, R. B. Morgunov, M. Gener, E. Canadell, R. P. Shibaeva and E. B. Yagubskii, Adv. Funct. Mater. 13403 (2003). 
[6] T. G. Prokhorova, L. I. Buravov, E. B. Yagubskii, L. V. Zorina, S. V. Simonov, V. N. Zverev, R. P. Shibaeva and E. Canadell, Eur. J. Inorg. Chem. 5611 (2015).

[7] A. D. Dubrovskii, N. G. Spitsina, L. I. Buravov, G. V. Shilov, O. A. Dyachenko, E. B. Yagubskii, V. N. Laukhin and E. Canadell, J. Mater. Chem. 151248 (2005).

[8] L. V. Zorina, S. S. Khasanov, S. V. Simonov, R. P. Shibaeva, V. N. Zverev, E. Canadell, T. G. Prokhorova and E. B. Yagubskii, Cryst. Eng. Comm. 132430 (2011).

[9] L. V. Zorina, S. S. Khasanov, S. V. Simonov, R. P. Shibaeva, P. O. Bulanchuk, V. N. Zverev, E. Canadell, T. G. Prokhorova and E. B. Yagubskii, Cryst. Eng. Comm. 14460 (2012).

[10] A. Audouard, J.-Y. Fortin, V. N. Laukhin, D. Vignolles, T. G. Prokhorova, E. B. Yagubskii and E. Canadell, Eur. Phys. J. B 87200 (2014).

[11] A. Audouard and J.-Y. Fortin, Low Temp. Phys. 40344 (2014), [Fiz. Nizk. Temp. 40444 (2014)].

[12] J.-Y. Fortin and A. Audouard, Phys. Rev. B 77134440 (2008).

[13] J.-Y. Fortin and A. Audouard, Phys. Rev. B 80214407 (2009).

[14] I. M. Lifschitz and A. M. Kosevich, Dokl. Akad. Nauk SSSR 96963 (1954).

[15] A. M. Kosevich and I. M. Lifschitz, Zh. Eks. Teor. Fiz. 29730 (1955).

[16] D. Shoenberg, Magnetic Oscillations in Metals (Cambridge University Press, Cambridge, 1984).

[17] R. G. Chambers, Proc. Phys. Soc. 88701 (1966).

[18] L. M. Falicov and H. Stachowiak, Phys. Rev. 147505 (1966).

[19] A. Bangura, A. Coldea, A. Ardavan, J. Singleton, A. Akutsu-Sato, H. Akutsu and P. Day, J. Phys. IV France 114285 (2004).

[20] S. S. Khasanov, B. Zh. Narymbetov, L. V. Zorina, L. P. Rozenberg, R. P. Shibaeva, N. D. Kushch, E. B. Yagubskii, R. Rousseau and E. Canadell, Eur. Phys. J. B 1419 (1998).

[21] A. Audouard, V. N. Laukhin, C. Proust, L. Brossard and N. D. Kushch, J. Phys. I France, 7 599 (1997).

[22] C. Proust, A. Audouard, V. N. Laukhin, L. Brossard, M. Honold, M. S. Nam, E. Haanappel, J. Singleton and N. D.Kushch, Eur. Phys. J. B 2131 (2001).

[23] R. B. Lyubovskii, S. I. Pesotskii, G. V. Shilov, E. I. Zhilyaeva, A. M. Flakina and R. N. Lyubovskaya, JETP Letters 98181 (2013).

[24] S. Uji and J. S. Brooks, The Physics of Organic Superconductors and Conductors, Springer 
Series Material Science Vol. 110 (Springer, 2008), p. 89.

[25] A. Audouard, J.-Y. Fortin, D. Vignolles, V. N. Laukhin, N. D. Kushch and E. B. Yagubskii, J. Phys.: Condens. Matter 28275702 (2016).

[26] D. Vignolles, A. Audouard, V. N. Laukhin, E. Canadell, T. G. Prokhorova and E. B. Yagubskii, Eur. Phys. J. B 71203 (2009).

[27] D. Vignolles, A. Audouard, V. N. Laukhin, E. Canadell, T. G. Prokhorova and E. B. Yagubskii, Synth. Met. 1602467 (2010).

[28] V. N. Laukhin, A. Audouard, D. Vignolles, E. Canadell, T. G. Prokhorova and E. B. Yagubskii, Low. Temp. Phys. 37749 (2011) [Fizika Nizkikh Temperatur 37943 (2011)].

[29] D. Vignolles, V. N. Laukhin, A. Audouard, T. G. Prokhorova, E. B. Yagubskii and E. Canadell, Eur. Phys. J. B 5153 (2006).

[30] A. Audouard, V. N. Laukhin, L. Brossard, T. G. Prokhorova, E. B. Yagubskii and E. Canadell, Phys. Rev. B 69144523 (2004).

[31] A. Audouard, V. N. Laukhin, J. Béard, D. Vignolles, M. Nardone, E. Canadell, and T. G. Prokhorova and E. B. Yagubskii, Phys. Rev. B 74233104 (2006).

[32] A. Coldea, A. Bangura, J. Singleton, A. Ardavan, A. Akutsu-Sato, H. Akutsu, S. S. Turner and P. Day, Phys. Rev. B 69085112 (2004).

[33] A. Bangura, A. Coldea, J. Singleton, A. Ardavan, A. Akutsu-Sato, H. Akutsu, S. S. Turner and P. Day, Phys. Rev. B 7214543 (2005). 